\title{
Estimate-Merge-Technique-based algorithms to track an underwater moving target using towed array bearing-only measurements
}

\author{
D V A N RAVI KUMAR ${ }^{1, *}$, S KOTESWARA RAO ${ }^{2}$ and K PADMA RAJU ${ }^{3}$ \\ ${ }^{1}$ Department of Electronics and Communications Engineering, Gayatri Vidya Parishad College of Engineering \\ for Women, Madhurawada, Visakhapatnam 530013, India \\ ${ }^{2}$ Department of Electronics and Communication Engineering, KL University, Guntur 522502, India \\ ${ }^{3}$ Department of Electronics and Communications Engineering, Jawaharlal Nehru Technological University, \\ Kakinada 533003, India \\ e-mail: ravikumardwarapu@gvpcew.ac.in; koteswararaosir1234@gmail.com; padmarajusir1234@gmail.com
}

MS received 12 August 2015; revised 11 June 2016; accepted 25 January 2017; published 4 July 2017

\begin{abstract}
Bearing-only passive target tracking is a well-known underwater defence issue dealt in the recent past with the conventional nonlinear estimators like extended Kalman filter (EKF) and unscented Kalman filter (UKF). It is being treated now-a-days with the derivatives of EKF, UKF and a highly sophisticated particle filter (PF). In this paper, two novel methods based on the Estimate Merge Technique are proposed. The Estimate Merge Technique involves a process of getting a final estimate by the fusion of a posteriori estimates given by different nonlinear estimates, which are in turn driven by the towed array bearing-only measurements. The fusion of the estimates is done with the weighted least squares estimator (WLSE). The two novel methods, one named as Pre-Merge UKF and the other Post-Merge UKF, differ in the way the feedback to the individual UKFs is applied. These novel methods have an advantage of less root mean square estimation error in position and velocity compared with the EKF and UKF and at the same time require much lesser number of computations than that of the PF, showing that these filters can serve as an optimal estimator. A testimony of the aforementioned advantages of the proposed novel methods is shown by carrying out Monte Carlo simulation in MATLAB R2009a for a typical war time scenario.
\end{abstract}

Keywords. Extended Kalman filter; unscented Kalman filter; particle filter; towed array; weighted least squares estimator.

\section{Introduction}

Under-water target tracking is a complex process, involving estimation of the position of a target and anticipation of its location at the future instants with minute errors. The essential ingredient of the tracking process is the measurements provided by a SONAR. They can be broadly classified into two types, namely active and passive measurements. In case of tracking with active measurements, the surveillance platform radiates emissions and captures the echo to get the information of range and bearing of the target. The process cannot be considered as a preferable one as it gives the information of the location and dynamics of the ownship to the enemy before the ownship gets the information of the foe. This problem is not associated with the SONAR passive measurements. Here no emissions will be made by the ownship's SONAR. It gets only the azimuth (bearing) information from the emissions generated by the propellers of the enemies' vehicle. The measurements

*For correspondence received by the SONAR are corrupted by the noise. This noisy information cannot be used for missile guidance. A tracking algorithm processes one of the two types of measurements or both to estimate the target motion parameters, namely range, bearing, course and velocity.

A lot of research was done and is being done in this area with an aim of developing an optimal tracking algorithm. Satisfactory results started after the famous work of Kalman [1] was published. Initially the conventional Kalman filter was applied for active tracking process with many assumptions, which led to the limitations for its usage in the real war environment. The most difficult feature that is assumed is as follows. The measurements are available in polar coordinates, which are converted to Cartesian form; thereafter the Kalman filter is applied. In this process the Gaussian noise with zero mean in polar coordinates is assumed to maintain the same statistics when converted to a Cartesian frame. This assumption is not considered by Lerro and Bar-Shalom [2], to make the Kalman filter suit the real time applications where the actual mean and covariance of noise in Cartesian frame are computed and 
this bias in mean is subtracted from the measurements in rectangular system to make the measurement errors to have a null mean.

The work of Lerro and Bar-Shalom [2] was extended by Suchomski [3], where exact expressions for bias and covariance corresponding to the three-dimensional case are computed. Here it is assumed that the elevation measurement is also available along with the azimuth and range.

The active tracking process is solved in a different way where the state equation is expressed in Cartesian coordinate system and the measurement equation (using measured range and bearing) is represented in the polar form, making the state equation a linear one and the measurement equation a nonlinear one. Thereafter, the nonlinear measurement equation is converted to a linear one with the help of a Taylor series expansion. This step is followed by the application of the conventional Kalman filter on the linear state and the measurement equations. The resulting filter is named as the extended Kalman filter (EKF) in the literature. The results are shown by Lerro and Bar-Shalom [2].

The EKF is used for passive tracking process in the same way as explained in the earlier paragraph with the only difference of taking into consideration the bearing measurements in the measurement vector instead of the range and the bearing measurements. The results are shown by Aidala and Hammel [4]. The afore-mentioned filter is named as Cartesian coordinate EKF in the literature, which exhibits unstable behaviour caused by the ill-conditioned covariance matrix, which in turn is caused by the coupling of the observable and unobservable components of the state vector. The decoupling of these components to attain stability is also shown by Aidala and Hammel [4], where the state vector comprises bearing rate, range rate to range ratio, bearing and reciprocal of the range instead of the conventional way of choosing the range and the velocity components. The resultant filter is called as the modified polar coordinate EKF.

The stability problem associated with the EKF is solved in a different way by Song and Speyer [5], where a modified gain function is introduced in the state vectors covariance matrix. This filter, called the modified-gain bearing-only EKF (MGBEKF), has shown a great promise for tracking application with bearing-only measurements. The modified gain function of Song and Speyer [5] is derived in a much simpler way by Galkowski and Islam [6]. This technique has the added advantage of causing less numerical difficulties.

Nardone et al [7] have shown how the bearing-only passive target tracking can be performed with the help of a pseudo-linear estimator (PLE) and the maximum likelihood estimator (MLE). The PLE is based on the technique of the least squares estimation, where the estimation is made in such a way that the mean square error in the estimation is minimum, whereas the MLE generates an estimate in such a way that the log likelihood function is maximized. The two algorithms of Nardone et al [7] used the batch processing, where all the previous measurements are required to generate the present estimate. The technique is hard to implement in the real time environment due to the huge memory requirements and large processing time. This problem is solved by Rao [8, 9], where the batch processing equations are converted to sequential equations to make them useful for the realistic situations. This is possible by updating the recursive SUMS whenever a new measurement is received without storing all the previous data. Rao [9] also showed that initialization of the estimate of the sequential MLE algorithm with PLE output instead of arbitrary values can yield much faster convergence. A similar technique is used by Rao [8], where the MGBEKF is initialized with the PLE output to yield superior results. The results given by Aidala and Nardone [10] have given a strong support to the ownship manoeuvring concept suggested by the researchers earlier primarily to maintain the observability when dealing with a single sensor. In that paper it is shown that the estimate bias associated with the long-range target tracking using PLE can be minimized to a great deal if the ownship continues manoeuvring.

A much simpler and accurate estimator called the unscented Kalman filter (UKF) is proposed by Julier and Uhlmann [11] to deal with the estimation problems associated with the nonlinear systems. Here the complex computations such as the calculation of the Jacobian and Hessian which are essential in EKF are avoided. This algorithm is applied to bearings only tracking (BOT) problem by Rao et al [12] to achieve good results. The key step employed here is the unscented transformation of the mean and the covariance over the nonlinear measurement equation associated with the passive target tracking process. Tracking a moving object in a prototype scenario using multiple sensors angle only measurements with UKF is performed by Zhang et al [13].

Some hybrid algorithms such as the one proposed by Rao et al [14] come up here and there; this shows the importance of tracking using bearing-only measurements. Some algorithms such as the one proposed by Zhou et al [15] used additional inputs (Doppler measurement) along with the conventional inputs (range and bearing) for tracking process to achieve superior results. Different kinds of particle filters (PFs) as given in Simon [16] and Ristick et al [17] were developed and made their way into almost all the engineering fields like BOT involving estimation problem. The PFs provide low estimation errors compared with all the existing stochastic algorithms at the cost of infeasibly high processor requirements and large computational time.

The discussion so far gives the information of all the estimators available in the literature that have contributed a lot for tracking an underwater moving target. It can be observed that the higher the processor requirements and complexity of technique, the better the performance of the estimators, and lesser the processor requirements, poorer the performance. At this point it is essential to design a filter that can provide a very good performance (low 
estimation error and low convergence time) at feasibly high processor requirements or in other words an optimal filter has to be designed. This is the motivation behind the present work.

Section 2 introduces the novel method of Estimate Merge Technique and explains the technical difference between the Pre-Merge and Post-Merge estimators. Section 3 gives mathematical modelling of a moving target, modelling of towed array measurements, mathematical expressions related to Estimate Merge Technique and performance metrics used to compare different nonlinear estimators. Section 4 deals with the simulation, analysis and results. Finally, the paper is concluded in section 5.

\section{Estimate Merge Technique}

The final estimate of the state of a moving target is obtained using the Estimate Merge Technique. The principle involved is to merge the estimates given by the individual estimators, which are driven by the towed array bearingonly measurements. The individual estimators can be any nonlinear estimators like UKF, which is used in this paper. The merger used in this particular case is the weighted least squares estimator (WLSE).

The two Estimate Merge Techniques proposed in this paper are named as Pre-Merge UKF and Post-Merge UKF; they differ in the way the feedback to the individual UKFs is supplied.

\subsection{Pre-Merge UKF}

In this case the individual UKFs generate their own feedback (the feedback signal is generated prior to the merging process). Based on the afore-mentioned mechanism it can be called as Pre-Merge UKF. A block diagram of PreMerge UKF is shown in figure 1.

\subsection{Post-Merge UKF}

If the individual UKFs are fed by the WLSE (the feedback signal is generated in a post-merging process) the corresponding algorithm is named as Post-Merge UKF. This technique can be well understood by observing the block diagram in figure 2.

In the block diagrams (figures 1 and 2) $Y 1(k), Y 2(k)$ are the measurements applied to the UKFs 1 and 2, respectively. $\hat{\mathbf{X}}(0 / 0), \mathbf{P}(0 / 0)$ are, respectively, the state and the covariance matrix with which the UKFs are initialized. Notations in the figure denote the estimate and covariance given by individual UKFs at time instant $k$ by considering the measurement at $k$ th instant. Finally, the estimated value and covariance of the proposed technique is denoted, respectively, by $\hat{\mathbf{X}}(k), \mathbf{P}(k)$.

\section{Mathematical modelling}

\subsection{Mathematical modelling of a moving target}

The state vector at time instant $k$, when tracking a moving target, comprises the position components $x(k), y(k)$ and the velocity components $\dot{x}(k), \dot{y}(k)$ :

$$
X(k)=\left[\begin{array}{llll}
x(k) & y(k) & \dot{x}(k) & \dot{y}(k)
\end{array}\right]^{\mathrm{T}} .
$$

The dynamics of a target moving with a constant velocity can be written as

$$
\left[\begin{array}{c}
x(k+1) \\
y(k+1) \\
\dot{x}(k+1) \\
\dot{y}(k+1)
\end{array}\right]=\left[\begin{array}{cccc}
1 & 0 & \Delta T & 0 \\
0 & 1 & 0 & \Delta T \\
0 & 0 & 1 & 0 \\
0 & 0 & 0 & 1
\end{array}\right]\left[\begin{array}{c}
x(k) \\
y(k) \\
\dot{x}(k) \\
\dot{y}(k)
\end{array}\right]+Q(k)
$$

The time span between the states is denoted by $\Delta T$. The process noise is zero-mean Gaussian with a covariance matrix as per Eq. (13) of Zhang et al [13] as follows:

$$
E\left[Q(k) Q(k)^{\mathrm{T}}\right]=\left[\begin{array}{cccc}
\Delta T^{3} / 3 & 0 & \Delta T^{2} / 2 & 0 \\
0 & \Delta T^{3} / 3 & 0 & \Delta T^{2} / 2 \\
\Delta T^{2} / 2 & 0 & \Delta T & 0 \\
0 & \Delta T^{2} / 2 & 0 & \Delta T
\end{array}\right] q
$$

where $q$ is the spectral density of the acceleration errors. Now Eq. (2) is rewritten as

$$
X(k+1)=F X(k)+Q(k)
$$

$F$ is called the state transition matrix and is given by $F=$ $\left[\begin{array}{rrrr}1 & 0 & \Delta T & 0 \\ 0 & 1 & 0 & \Delta T \\ 0 & 0 & 1 & 0 \\ 0 & 0 & 0 & 1\end{array}\right]$

\subsection{Mathematical modelling of towed array measurements}

Let $(S 1(1), S 1(2)),(S 2(1), S 2(2))$ be the coordinates of two passive sensors $\mathrm{S} 1$ and $\mathrm{S} 2$ placed on the towed array and $(x(k), y(k))$ represent the coordinate pair at time ' $k$ ' of the target that is to be tracked. Now with the help of figure 3 the actual azimuth or the bearing at the two sensors S1 and $\mathrm{S} 2$ can be written as

$$
\begin{aligned}
& B 1(k)=\tan ^{-1}\left(\frac{y(k)-S 1(2)}{x(k)-S 1(1)}\right), \\
& B 2(k)=\tan ^{-1}\left(\frac{y(k)-S 2(2)}{x(k)-S 2(1)}\right) .
\end{aligned}
$$

The corresponding noise-corrupted bearing measurements at $\mathrm{S} 1$ and $\mathrm{S} 2, B m 1(k), B m 2(k)$, can be written as 


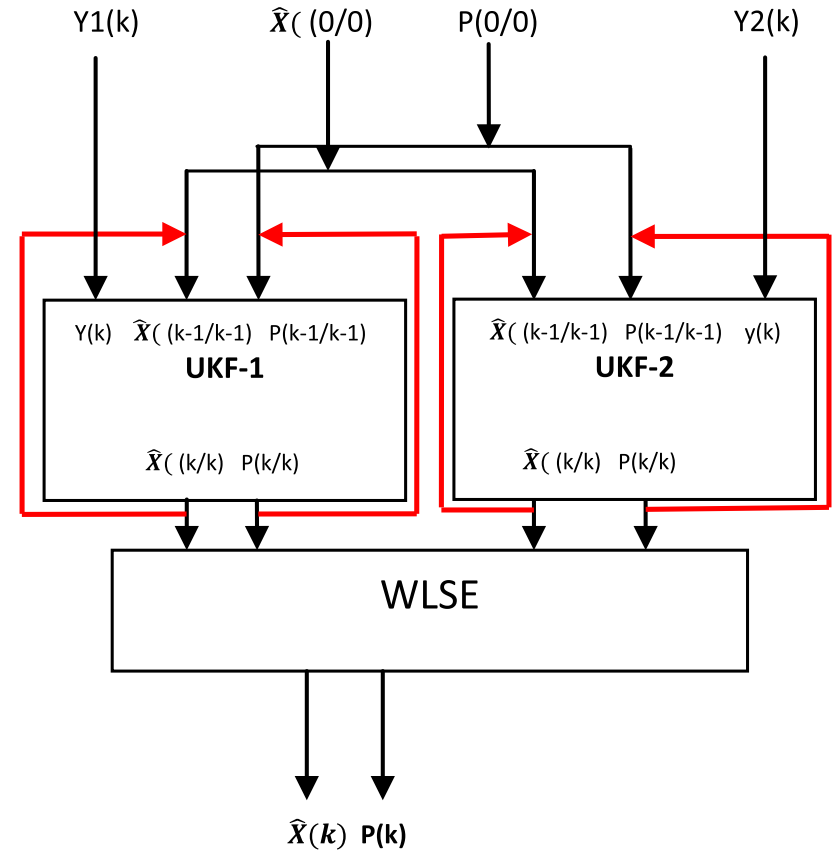

Figure 1. Pre-Merge UKF.

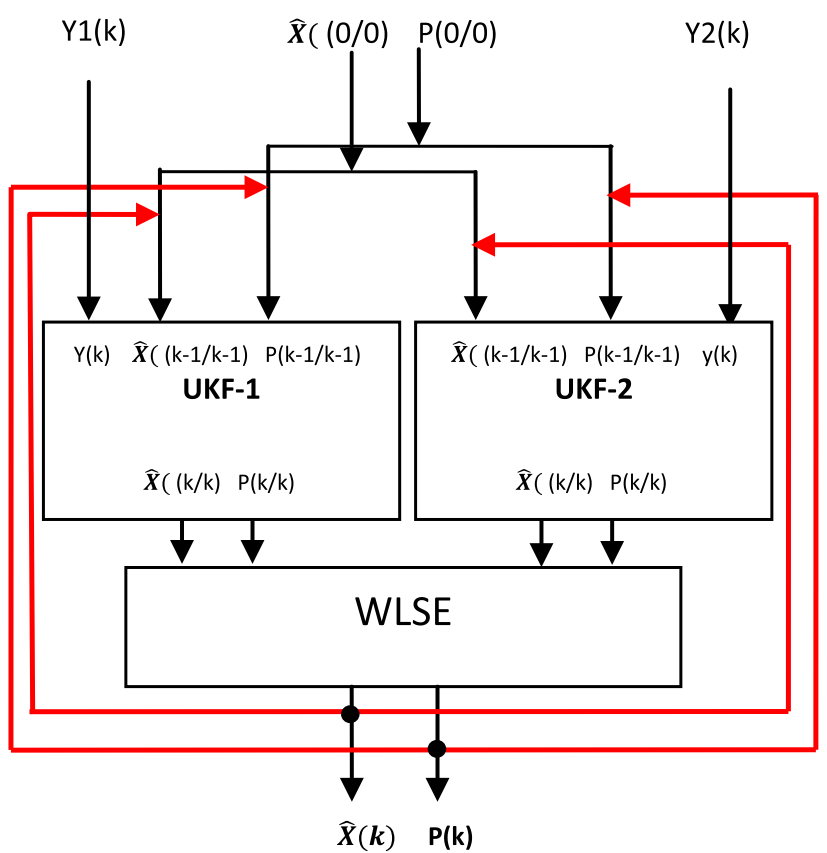

Figure 2. Post-Merge UKF.

$$
B m 1(k)=B 1(k)+B n 1(k) \text { and } B m 2(k)=B 2(k)+B n 2(k)
$$

$B n 1(k), B n 2(k)$ are the Gaussian noises added to the bearings $B 1(k), B 2(k)$.

The measurement equation that expresses the measurements in terms of the state vector can be written as

$$
\begin{gathered}
\qquad y(k)=h(x(k), k)+v(k) \\
\text { With the measurement vector } y(k)=\left[\begin{array}{l}
B m 1(k) \\
B m 2(k)
\end{array}\right] \\
\text { transfer function } h(x(k), k)=\left[\begin{array}{l}
\tan ^{-1}\left(\frac{y(k)-S 1(2)}{x(k)-S 1(1)}\right) \\
\tan ^{-1}\left(\frac{y(k)-S 2(2)}{x(k)-S 2(1)}\right)
\end{array}\right]
\end{gathered}
$$

$v(k)$ represents the sensor noise of Gaussian type with zero mean and co-variance $R$ :

$$
R=E\left[v(k) v(k)^{\mathrm{T}}\right]=\left[\begin{array}{cc}
\sigma_{1}^{2} & 0 \\
0 & \sigma_{2}^{2}
\end{array}\right]
$$

where $\sigma_{1}^{2}, \sigma_{2}^{2}$ are the variances of noise in the measurements given by the sensors $\mathrm{S} 1, \mathrm{~S} 2$, respectively.

\subsection{Estimate Merge Technique}

With the help of the sensor pair $\mathrm{S} 1, \mathrm{~S} j+1$, the measurement $y m j(k)$ can be obtained using Eq. (7). The same concept is extended to all the $p+1$ sensors of the towed array to obtain $y m 1(k), y m 2(k), \ldots, y m p(k)$. The related transfer functions $h 1(x(k), k), h 2(x(k), k), \ldots, h p(x(k), k)$ can be derived from Eq. (8), while the corresponding co-variance matrices $R 1$, $R 2, \ldots, R p$, can be obtained from Eq. (9).The $p$ different UKFs process these $p$ measurements to yield $p$ estimates of the state of a moving target, namely $\hat{X} 1\left(\frac{k}{k}\right), \hat{X} 2\left(\frac{k}{k}\right), \ldots, \hat{X} p\left(\frac{k}{k}\right)$, with the corresponding covariance matrices $P 1(k / k), P 2(k / k), \ldots, P p(k /$ $k$ ), where $\hat{X} j(k / k)$ is a matrix of the following form:

$$
\hat{X} j(k / k)=\left[\begin{array}{llll}
\hat{X}_{(1)}(k / k) & \hat{X} j_{(2)}(k / k) & \ldots & \hat{X} j_{(n)}(k / k)
\end{array}\right]^{\mathrm{T}} .
$$

$\hat{X} j_{(i)}(k / k)$ is the estimate of $i$ th element (of $n$ elements of state vector) given by the $j$ th $\mathrm{UKF}$ (of $p \mathrm{UKFs}$ ); $P j(k / k)$ is a matrix of the following form:

$$
P j\left(\frac{k}{k}\right)=\operatorname{diag}\left[\begin{array}{llll}
p_{j 1} & p_{j 2} & \ldots & p_{j n}
\end{array}\right] .
$$

$p_{j i}$ is the variance in the estimate of $i$ th element of state vector $X$ given by the $j$ th UKF.

The relation between the $i$ th element of merged estimate $X_{(i)}(k / k)$ and the $i$ th element of the estimates given by $p$ UKFs $\left(\hat{X} 1_{(i)}(k / k), \hat{X} 2_{(i)}(k / k), \ldots, \hat{X} p_{(i)}(k / k)\right)$ is given by

$$
\left[\begin{array}{c}
\hat{X} 1_{(i)}(k / k) \\
\hat{X} 2_{(i)}(k / k) \\
\cdot \\
\hat{X} p_{(i)}(k / k)
\end{array}\right]=\left[\begin{array}{c}
1 \\
1 \\
\cdot \\
\cdot \\
1
\end{array}\right]_{p x 1} X_{(i)}\left(\frac{k}{k}\right)+l_{i}(k) .
$$




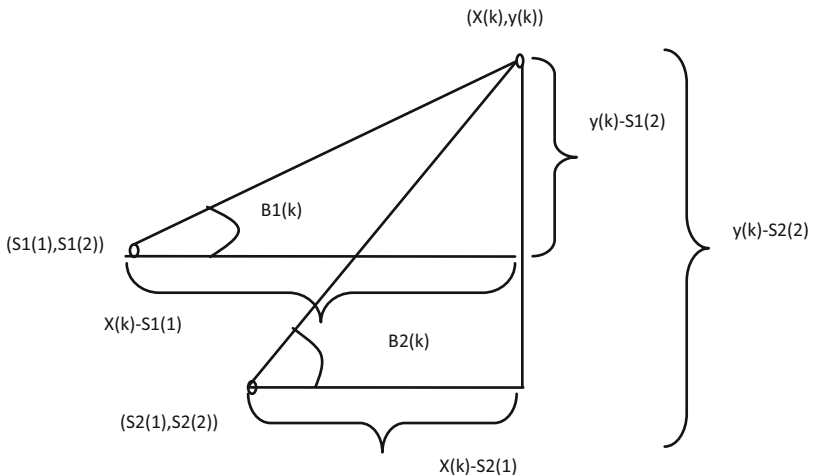

Figure 3. Bearing calculation using sensor and target coordinates.

In this discussion it is assumed that all the measurements have equal weight.

Here $l_{i}(k)$ is a $p \times 1$ matrix of Gaussian noise in the $i$ th element of the estimate given by the $p$ UKFs with zero mean and covariance matrix $R_{(i)}$ :

$$
R_{(i)}=\left[\begin{array}{cccc}
p_{1 i} & 0 & . & \cdot \\
0 & p_{2 i} & 0 & \cdot \\
. & \cdot & \cdot & \cdot \\
0 & 0 & \cdot & p_{p i}
\end{array}\right] .
$$

Equation (13) is obtained from Eq. (11). Now, Eq. (12) can be rewritten as

$$
\begin{gathered}
Y_{(i)}(k / k)=H X_{(i)}(k / k)+l_{i}(k) \\
\text { with } Y_{(i)}\left(\frac{k}{k}\right)=\left[\begin{array}{c}
\hat{X} 1_{(i)}\left(\frac{k}{k}\right) \\
\hat{X} 2_{(i)}\left(\frac{k}{k}\right) \\
\vdots \\
\hat{X} p_{(i)}\left(\frac{k}{k}\right)
\end{array}\right] .
\end{gathered}
$$

The estimate of $X_{(i)}(k / k)$ in Eq. (14), now denoted by $\hat{X}_{(i)}(k / k)$, is computed in such a way that the mean square error in the estimation process is minimum, i.e., following the LSE algorithm. The solution as per Eq. (3.15) of Simon [16] is in the following form:

$$
\hat{X}_{(i)}(k / k)=\left(H^{T} R_{(i)}^{-1} H\right)^{-1} H^{T} R_{(i)}^{-1} Y_{(i)}\left(\frac{k}{k}\right) .
$$

Using Eq. (3.19) of Simon [16], Eq. (17) is simplified to Eq. (18):

$$
\hat{X}_{(i)}\left(\frac{k}{k}\right)=\left(\sum_{j=1}^{p} \frac{1}{p_{j i}^{2}}\right)^{-1}\left(\sum_{j=1}^{p} \frac{X j_{(i)}\left(\frac{k}{k}\right)}{p_{j i}^{2}}\right) .
$$

The final estimate of the targets state or the final merged estimate at $k$ th instant considering $k$ th measurement is given as follows:

$$
\hat{X}\left(\frac{k}{k}\right)=\left[\hat{X}_{(1)}\left(\frac{k}{k}\right) \hat{X}_{(2)}\left(\frac{k}{k}\right) \ldots \hat{X}_{(n)}\left(\frac{k}{k}\right)\right]^{T} .
$$

The corresponding covariance is given by

$$
P(k / k)=\frac{1}{p} \sum_{i=1}^{p} P i\left(\frac{k}{k}\right)
$$

3.3a Pre- and Post-Merge UKF: In the Pre-Merge UKF, individual estimators generate their own feedback (priormerging process), while in Post-Merge UKF the WLSE supplies them (post-merging process). The mathematical expressions related to these techniques and the difference between these two algorithms can be well understood by observing table 1 .

\subsection{Performance metrics to compare the different nonlinear estimators}

3.4a RMS position error (in metres): As per Eq. (6.104) of Ristick et al [17] the RMS position error at $k$ th instant of time considering $N$ Monte Carlo runs is given by the relation

$$
\begin{aligned}
& \text { RMS position error }(k) \\
& =\sqrt{\frac{1}{N} \sum_{i=1}^{N}\left(x_{i}(k)-\hat{x}_{i}(k)\right)^{2}+\left(y_{i}(k)-\hat{y}_{i}(k)\right)^{2}}
\end{aligned}
$$

where $x_{i}(k), y_{i}(k)$ are the true position components of the target in $x$ and $y$ directions, respectively, at the $k$ th instant and $i$ th Monte Carlo run, while $\hat{x}_{i}(k), \hat{y}_{i}(k)$ are the estimated values.

\section{4b RMS velocity errors (in $\mathrm{m} / \mathrm{s}$ ):}

$R M S$ velocity error $(k)$

$$
=\sqrt{\frac{1}{N} \sum_{i=1}^{N}\left(\dot{x}_{i}(k)-\hat{x}_{i}(k)\right)^{2}+\left(\dot{y}_{i}(k)-\hat{y}_{i}(k)\right)^{2}} .
$$

Similar to Eq. (21), $\dot{x}_{i}(k), \dot{y}_{i}(k)$ denote actual velocity components, while $\hat{x}_{i}(k), \hat{y}_{i}(k)$ represent the estimated velocities.

$3.4 \mathrm{c}$ Convergence time (in seconds): It is the time instant after which the RMS position error is less than $300 \mathrm{~m}$.

\section{Simulation, analysis and results}

A typical wartime scenario considered for the evaluation of the algorithm is as follows. 
Table 1. Pre- Merge and Post-Merge UKF algorithm comparision.

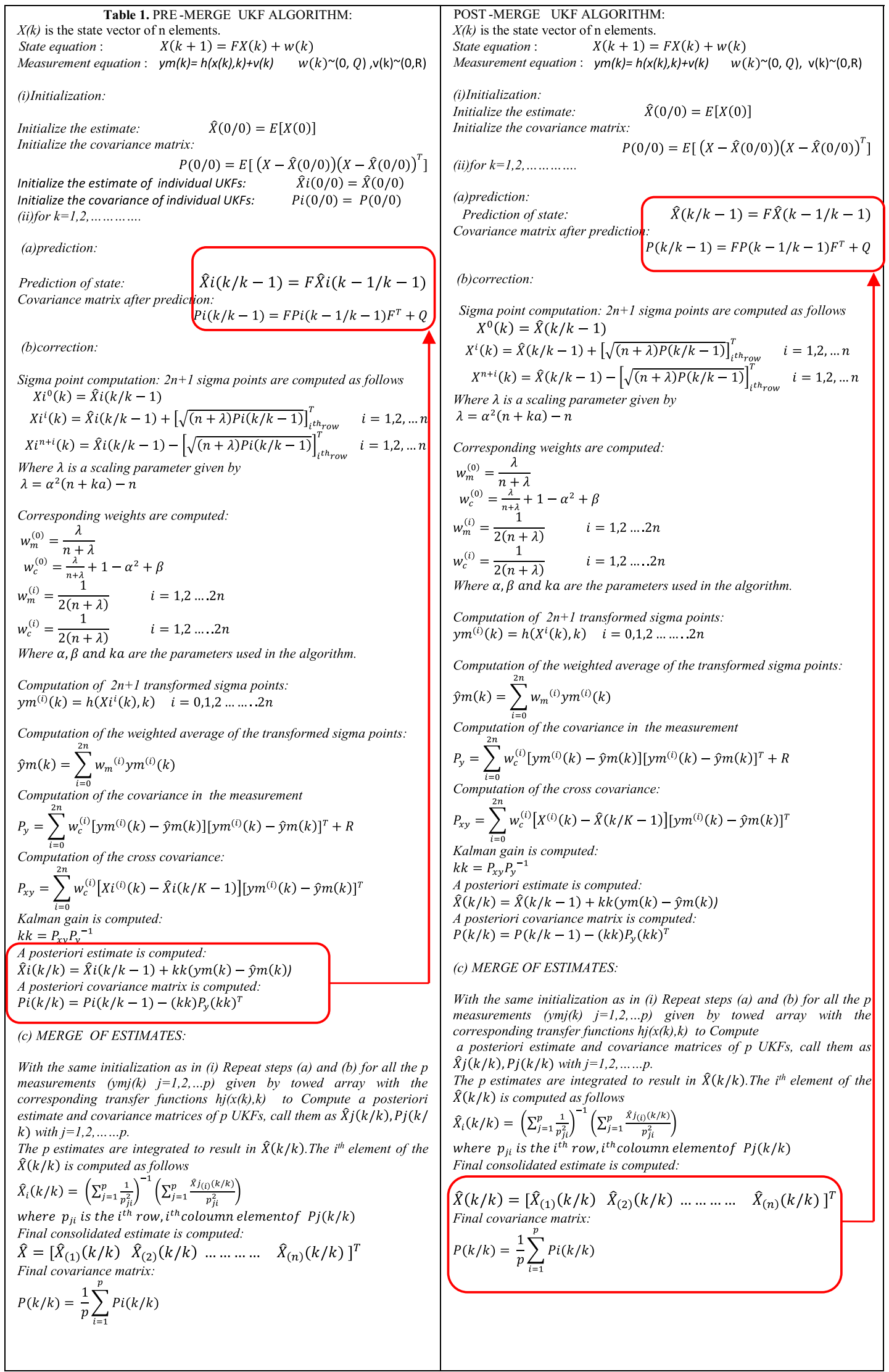




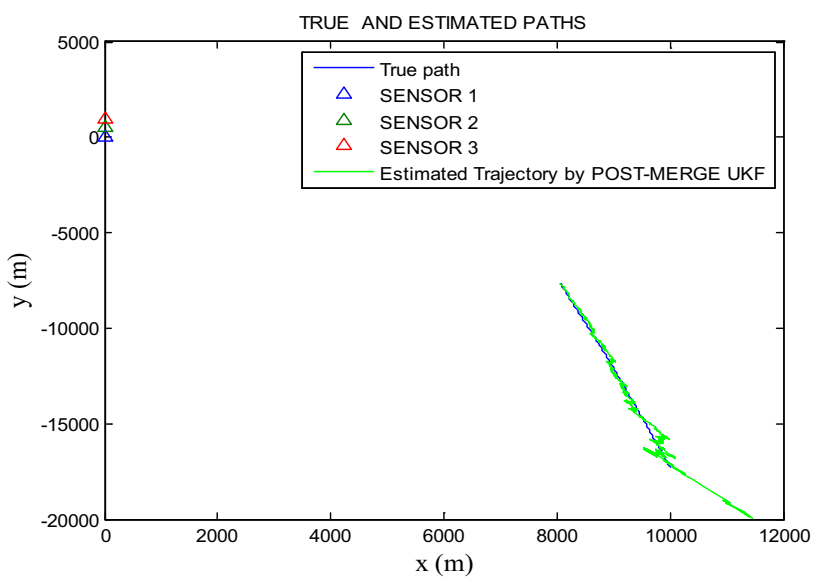

Figure 4. Actual and estimated trajectory of the target.

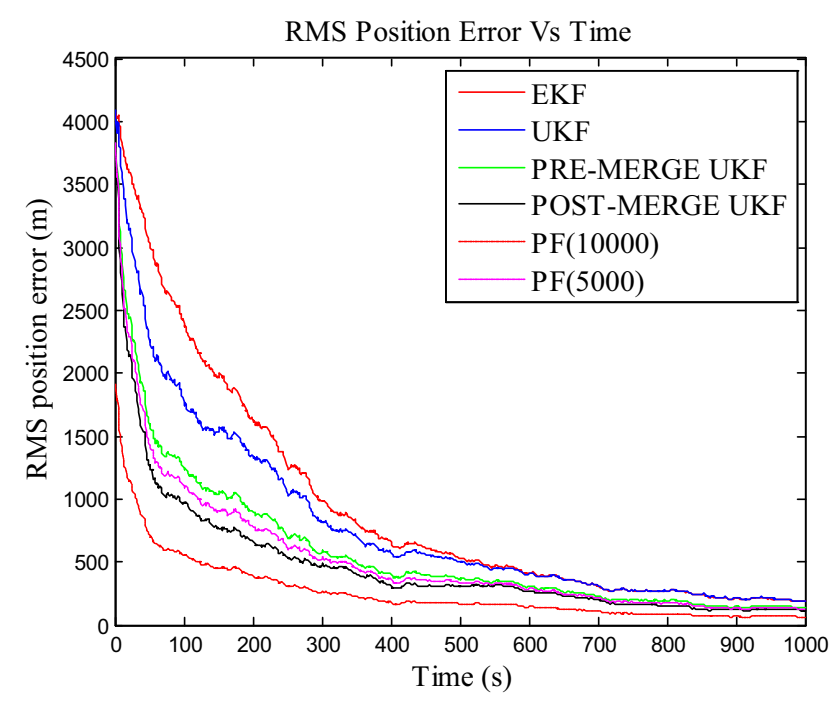

Figure 5. RMS position error vs time.

Target parameters The target is initially at a distance of $20 \mathrm{~km}$ and a bearing of $300^{\circ}$ with respect to positive $x$-axis $\left(150^{\circ}\right.$ with respect to the true north). The target moves with a constant course of $100^{\circ}$ and a velocity of $10 \mathrm{~m} / \mathrm{s}$. The acceleration errors in $x$ and $y$ directions are Gaussian with zero mean and a standard deviation of $0.01 \mathrm{~m} / \mathrm{s}^{2}$.

Ownship parameters The ownship is positioned at the origin and equipped with a towed array that comprises three sensors $\mathrm{S} 1, \mathrm{~S} 2$ and $\mathrm{S} 3$ at the locations $(0,0),(0,500 \mathrm{~m})$ and $(0,1000 \mathrm{~m})$. The SONAR of the ownship operates in the passive mode and hence the three sensors of the towed array capture the bearing measurements of the radiating target only after every $1 \mathrm{~s}$. The measurements are corrupted by a Gaussian noise of $0.28^{\circ}$ standard deviation and 0 mean. The simulation is carried out for $1000 \mathrm{~s}$.

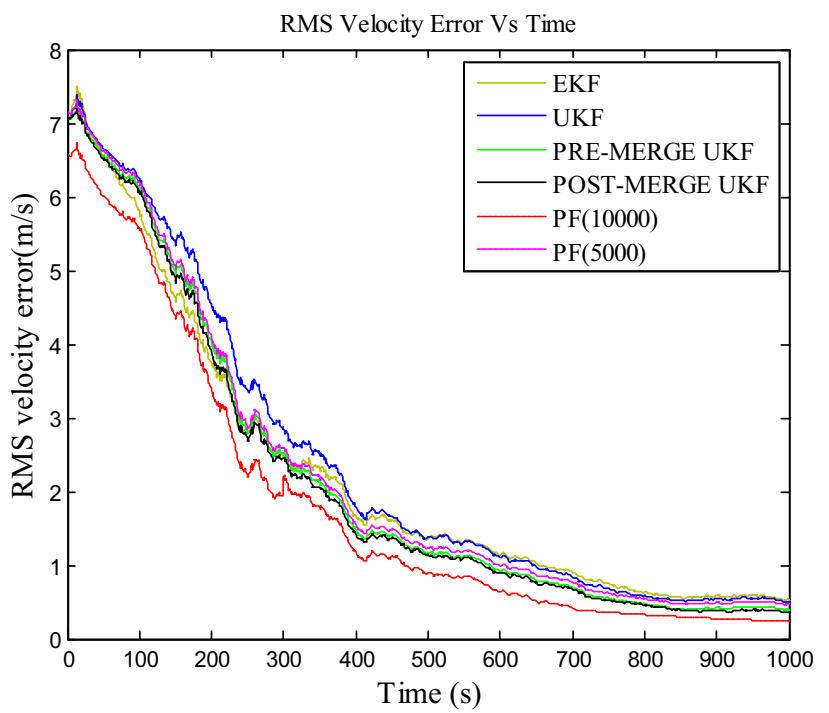

Figure 6. RMS velocity error vs time.

Table 2. Comparison of the existing and proposed nonlinear estimators.

\begin{tabular}{lccc}
\hline Algorithm & $\begin{array}{c}\text { RMS error in } \\
\text { velocity (m/s) }\end{array}$ & $\begin{array}{c}\text { RMS error in } \\
\text { position }(\mathrm{m})\end{array}$ & $\begin{array}{c}\text { Time of } \\
\text { convergence } \\
(\mathrm{s})\end{array}$ \\
\hline EKF & 0.5342 & 187 & 704 \\
UKF & 0.5053 & 187 & 704 \\
Pre-Merge UKF & 0.3952 & 134 & 596 \\
Post-Merge UKF & 0.3629 & 115 & 403 \\
PF(5000) & 0.4652 & 124 & 588 \\
PF(10000) & 0.2392 & 62 & 277 \\
\hline
\end{tabular}

Filter parameters The two UKFs used are initialized with an estimate of $\hat{X}(0 / 0)=[13000,-20000,3,5]^{\mathrm{T}}$ and a covariance of $P(0 / 0)=\operatorname{diag}\left[\begin{array}{llll}9000000 & 9000000 & 25 & 25\end{array}\right]$. The transformation parameter $\alpha$ is chosen as $0 ; \beta$ is set to 2 , which is an optimal value when dealing with Gaussians; $k a$ is set to zero. The weights of WLSE used for merging of estimates are taken as equal without any bias. The PF used for comparison uses adaptive resampling and the numbers of particles considered are 5000 and 10000. The total number of Monte Carlo simulations considered for the evaluation of the proposed algorithm is 50 .

Figure 4 shows how the estimated trajectory given by the Estimate-Merge-Technique-based algorithm catches up with the actual trajectory as time progresses. This illustrates the success of the proposed technique.

The estimation error (RMS position error and RMS velocity error) and time of convergence of the proposed techniques (especially Post-Merge UKF) are lower than 
Table 3. Computational complexity and processing time of various nonlinear estimators.

\begin{tabular}{lcc}
\hline Algorithm & $\begin{array}{c}\text { Computational complexity (no. of } \\
\text { sigma points) }\end{array}$ & $\begin{array}{c}\text { Processing } \\
\text { time (ms) }\end{array}$ \\
\hline EKF & 1 & 0.02 \\
UKF & 9 & 0.18 \\
Pre-Merge & 18 & 0.36 \\
$\quad$ UKF & 18 & 0.36 \\
Post-Merge & & \\
$\quad$ UKF & 5000 & 100 \\
PF(5000) & 10000 & 200 \\
PF(10000) & & \\
\hline
\end{tabular}

those of the existing algorithms like EKF, UKF and PF using 5000 particles $(\mathrm{PF}(5000))$ as shown in figures 5 and 6 and table 2. This superiority is due to the fact that the collective opinion about the state of a moving target is much better than the individual opinions given by a set of UKFs. Among the two Merge-Techniquebased algorithms the performance of the Post-Merge UKF is better than that of the Pre-Merge UKF. The reason for this is that during each iteration a better estimate (merged estimate provided by WLSE) is fed back to the individual UKFs in case of Post-Merge UKF as compared with the Pre-Merge UKF technique. However, the PF using 10000 particles $(\mathrm{PF}(10000))$ exhibits slightly upper hand over the novel techniques in terms of the estimation errors. However, PF(10000) struggles to work properly in the tough real world conditions due to its computational complexity and high processing times.

The computational complexity (based on the number of sigma points used in the implementation) of the various algorithms is given in table 3 . The computational time required to process the measurements on an Intel core $^{\mathrm{TM}}$ i7-3970X 3.5-GHz processor is also included in the table.

The numbers in table 3 clearly indicate that the computational complexity of the proposed methods is nearly
Table 5. Estimation error in position of different estimators at various initial ranges of the target.

\begin{tabular}{lcccc}
\hline & $\begin{array}{c}\text { Sc-1, } \\
\text { Algorithm }\end{array}$ & $\begin{array}{c}\text { Sc-2, } \\
R=4500\end{array}$ & $\begin{array}{c}\text { Sc-3, } \\
R=5000\end{array}$ & $\begin{array}{c}\text { Sc-4, } \\
R=10000\end{array}$ \\
\hline EKF & 638 & 485 & 488 & 699 \\
UKF & 232 & 212 & 452 & 742 \\
Pre-Merge & 139 & 135 & 309 & 515 \\
$\quad$ UKF & & & & \\
Post-Merge & 109 & 123 & 255 & 425 \\
$\quad$ UKF & & & & \\
PF(5000) & 139 & 137 & 280 & 483 \\
PF(10000) & 41 & 44 & 162 & 307 \\
\hline & & & &
\end{tabular}

equal to that of EKF and UKF, 250 times less than that of $\mathrm{PF}(5000)$ and 500 times less than that of $\mathrm{PF}(10000)$. This in turn reflects on the processing time $\left(T_{\mathrm{p}}\right)$, which is apparent from the table. The Post-Merge technique takes almost the same time as that of EKF, UKF and PreMerging method, and its value $(0.36 \mathrm{~ms})$ is much lower than that of $\mathrm{PF}(5000)(100 \mathrm{~ms})$ and $\mathrm{PF}(10000)(200 \mathrm{~ms})$. In real time, for a robust algorithm, the processing time should be much less than the time interval between the measurements, i.e., $T_{\mathrm{p}}<=10 \%$ of $\Delta T$. This condition ensures that the previous measurements are processed much before the successive measurements are received. This condition is satisfied for all the algorithms except for the case of PF, which makes its importance a questionable one in real time despite having low estimation errors.

From this discussion it can be concluded that the proposed method produces reasonably low estimation bugs and the implementation can be done at acceptably high processing times. In other words, we can say that the Estimate-Merge-Technique-based algorithms can serve as optimal filters for underwater defence issue like BOT.

A rigorous simulation is carried out to show the robustness of the novel methods. The algorithms are tested in nine

Table 4. Different scenarios considered to evaluate the performance of the proposed technique.

\begin{tabular}{|c|c|c|c|c|c|c|c|c|c|c|}
\hline & Sc-1 & & Sc-2 & Sc-3 & Sc-4 & Sc-5 & Sc-6 & Sc-7 & Sc-8 & Sc-9 \\
\hline$R(m)$ & 4500 & & 5000 & 10000 & 15000 & - & - & - & - & - \\
\hline$B(\operatorname{deg})$ & 300 & & - & - & - & - & - & - & - & - \\
\hline$T_{\mathrm{cr}}(\mathrm{deg})$ & 315 & & - & - & - & 0 & - & - & 270 & - \\
\hline$V(\mathrm{~m} / \mathrm{s})$ & 10 & & - & - & - & - & - & - & - & - \\
\hline Sigma $B(\operatorname{deg})$ & 0.28 & & - & - & - & 0.28 & 0.57 & 1.14 & 0.28 & - \\
\hline$X(0 / 0)$ error & {$\left[\begin{array}{c}+3000 \\
-3000 \\
+5 \\
-5\end{array}\right]$} & & - & - & - & - & - & - & {$\left[\begin{array}{c}+3000 \\
-3000 \\
+5 \\
-5\end{array}\right]$} & {$\left[\begin{array}{c}+10000 \\
-3000 \\
+7 \\
-5\end{array}\right]$} \\
\hline$P(0 / 0)$ & $\operatorname{Diag}[9000000 \quad 9000000$ & $25 \quad 25]$ & - & - & - & - & - & - & - & - \\
\hline
\end{tabular}

In the table indicates that the value in the grid is the same as the value in the immediate left grid. The numbers are not directly entered in the table to indicate the primary difference between the scenarios. The filter and other parameters are the same as mentioned earlier 

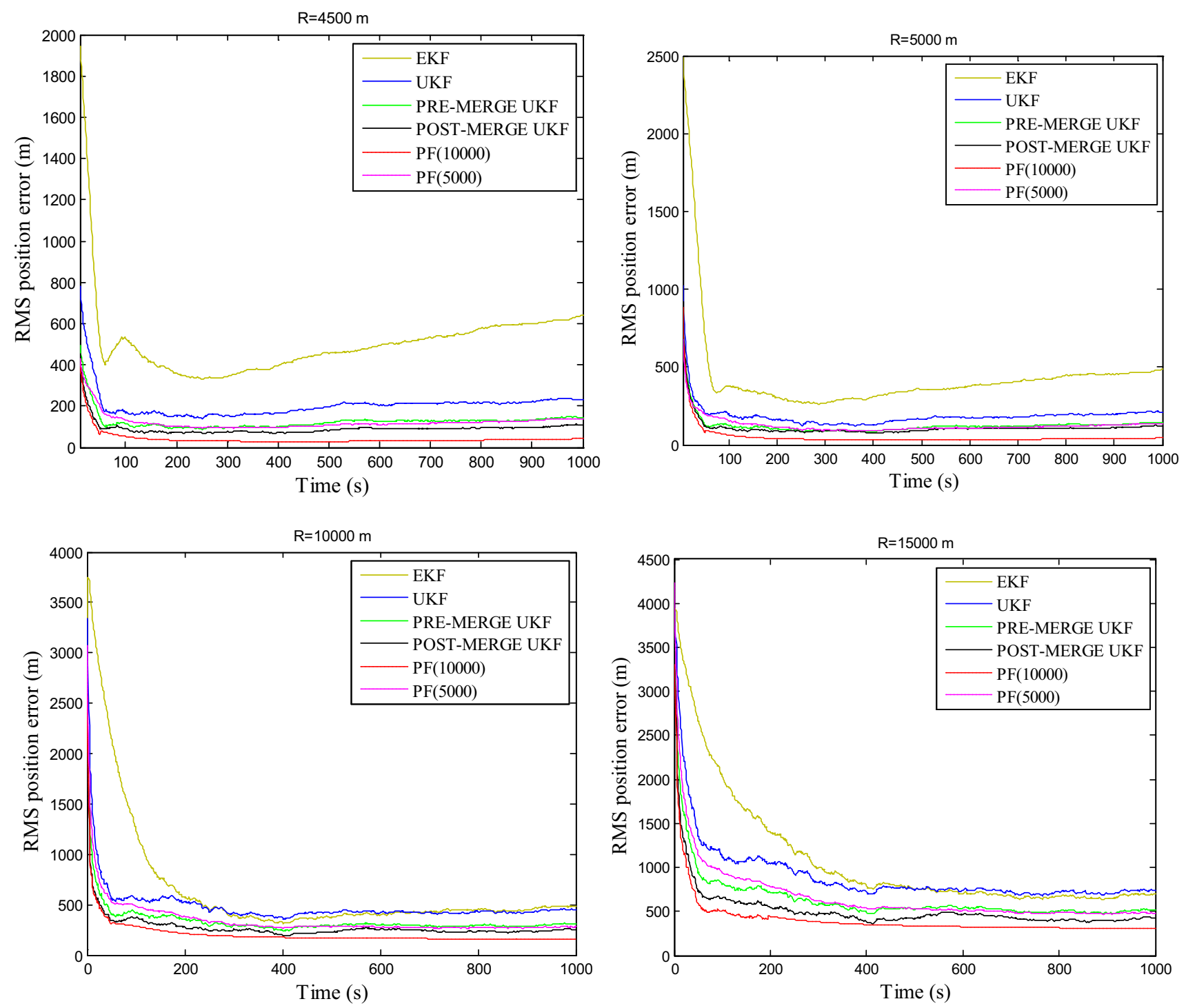

Figure 7. RMS position error vs time of different estimators at initial range $R=4500 \mathrm{~m}, R=5000 \mathrm{~m}, R=10000 \mathrm{~m}$ and $R=15000 \mathrm{~m}$.

different scenarios $(\mathrm{Sc}-1, \mathrm{Sc}-2, \ldots, \mathrm{Sc}-9)$ as given in table 4 to study the behaviour of all the algorithms with varying range, varying noise intensities and the effect of poor and good estimator initializations.

\subsection{Performance of algorithms with varying range}

Scenarios Sc-1-Sc-4 are considered to evaluate the performance of the proposed and the existing algorithms with varying initial ranges of the target.

Table 5 and figure 7 show that the proposed method exhibits very good performance while tracking targets in all the ranges; at the same time some algorithms like EKF struggle to track properly over low ranges. The reason for this is that the degrees of nonlinearites are more severe over
Table 6. Estimation error in position of different estimators at various bearing noise intensities.

\begin{tabular}{lccc}
\hline Algorithm & $\begin{array}{c}\text { Sc-5 } \\
\text { sd }=0.28^{\circ}\end{array}$ & $\begin{array}{c}\text { Sc-6 } \\
\text { sd }=0.57^{\circ}\end{array}$ & $\begin{array}{c}\text { Sc-7 } \\
\text { sd }=1.14^{\circ}\end{array}$ \\
\hline EKF & 373 & 723 & 1695 \\
UKF & 350 & 684 & 1695 \\
Pre-Merge UKF & 254 & 472 & 1115 \\
Post-Merge UKF & 216 & 372 & 787 \\
PF(5000) & 219 & 451 & 969 \\
PF(10000) & 110 & 176 & 582 \\
\hline
\end{tabular}

low ranges. These nonlinearities are linearized in EKF, which affects the innovation term and the covariance, which inturn affects the gain computations and hence 

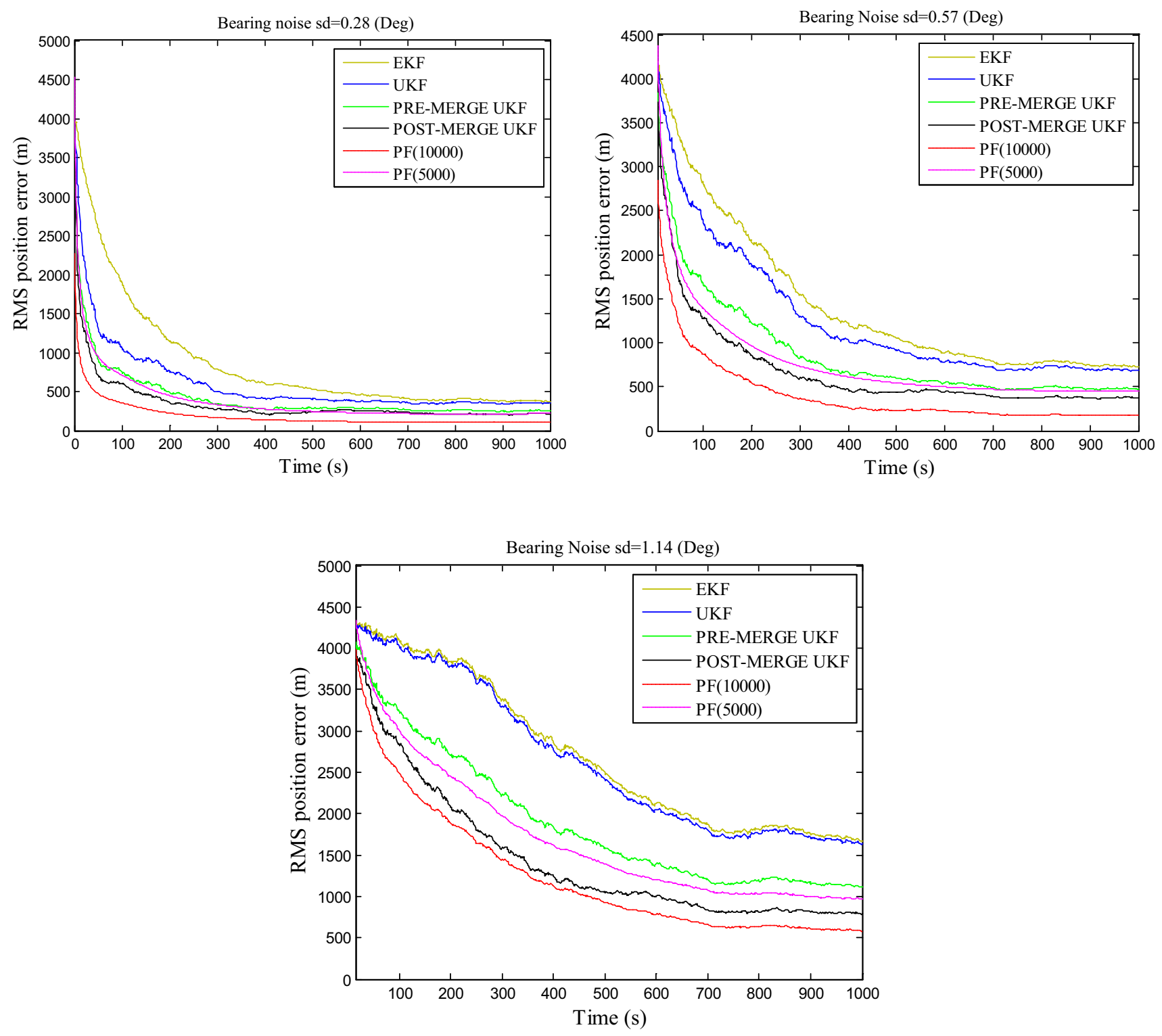

Figure 8. RMS position error vs time of different estimators at bearing noise intensities $\mathrm{sd}=0.28^{\circ}, \mathrm{sd}=0.57^{\circ}$ and $\mathrm{sd}=1.14^{\circ}$.

produces more estimation errors in the state estimate. Secondly, the posterior pdf becomes more non-Gaussian at high nonlinearities. It is treated as purely Gaussian, which causes even more estimation errors.

\subsection{Performance of algorithms with varying noise intensity}

Scenarios Sc-5-Sc-7 are considered to evaluate the performance of the proposed and the existing algorithms with varying bearing noise intensities.

Table 6 and figure 8 show that the introduced novel methods exhibit consistently good performance even under various noise conditions.

\subsection{Performance of algorithms with good and poor initializations}

Scenarios Sc-8 and Sc-9 are considered to evaluate the performance of the proposed and the existing algorithms with good and poor estimator initializations.

Table 7 and figure 9 clearly show that the performance of the proposed method does not suffer much by poor initialization. On the other hand, the estimators like EKF diverge if the algorithm is not initialized properly. This is because of picking a bad operating point (poor initialization) about which we have carried out the linearization. This problem is dealt with using the concept of unscented transformation of sigma points in the other algorithms. It is also seen that for poor initilization the errors gradually 
Table 7. Estimation error in position of different estimators with good and poor initializations.

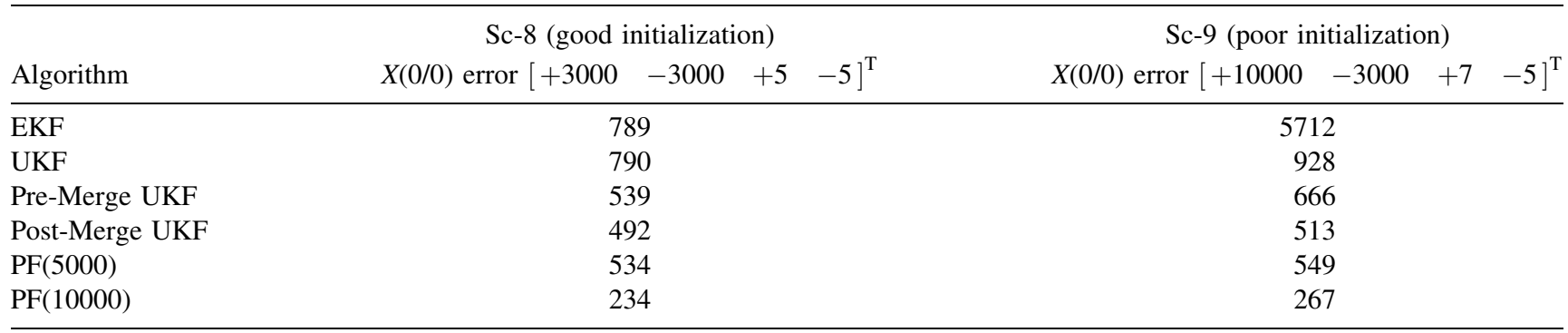
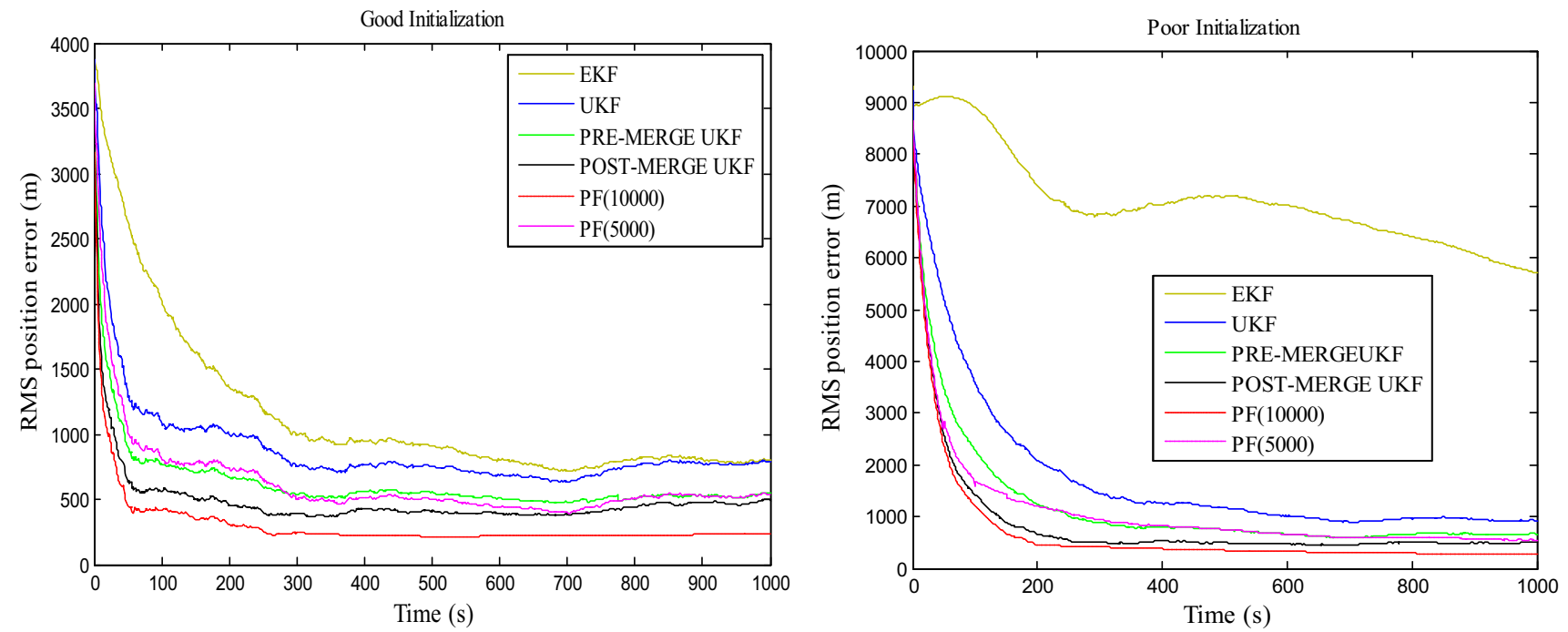

Figure 9. RMS position error vs time of different estimators with good and poor initializations.

reduce while they fall abruptly in case of good initialization for all the algorithms except EKF.

\section{Conclusions}

1. The estimation error associated with the proposed Estimate-Merge-Technique-based algorithms is much smaller than those achieved with the conventional nonlinear estimators like the EKF and UKF.

2. The computational complexity (processor requirements) and processing time associated with the novel method are slightly more than those of the EKF, UKF and much lesser than those of PF.

From these two points, it can be concluded that for a real time estimation problem like bearing-only passive target tracking, optimal results (acceptably low estimation error and feasibly high processor requirements) can be achieved with the proposed algorithms. The rigorous simulation also indicates that the superiority of the proposed method holds good under varying conditions of initial range, noise intensity and even under poor initialization scenarios. The performance of all the existing nonlinear estimators can be transformed to a higher level if they are coupled with the proposed algorithms.

\section{Acknowledgement}

The first author would like to thank the management of Gayatri Vidya Parishad College of Engineering for Women, Madhurawada, Visakhapatnam 530013, for providing all the resources essential for smooth completion of the present work. The authors would also like to thank the editors and reviewers, who have all given valuable suggestions to make the present work more worthy.

\section{References}

[1] Kalman R E 1960 A new approach to linear filtering and prediction problems. Trans. ASME_J. Basic Eng. 82(Ser. D): $35-45$

[2] Lerro D and Bar-Shalom Y 1993 Tracking with debiased consistent converted measurements versus EKF. IEEE Trans. Aerosp. Electron. Syst. 29(3): 1015-1022 
[3] Suchomski P 1999 Explicit expressions for debiased statistics of 3D converted measurements. IEEE Trans. Aerosp. Electron. Syst. 35(1): 368-370

[4] Aidala V J and Hammel S E 1983 Utilization of modified polar coordinates for bearings only tracking. IEEE Trans. Autom. Control 28(3): 283-294

[5] Song T L and Speyer J L 1985 A stochastic analysis of a modified gain extended Kalman filter with applications to estimation with bearings-only measurements. IEEE Trans. Autom. Control 30(10): 940-949

[6] Galkowski P J and Islam M A 1991 An alternative derivation of the modified gain function of Song and Speyer. IEEE Trans. Autom. Control 36(11): 1323-1326

[7] Nardone S C, Lindgren A G and Gong K F 1984 Fundamental properties and performance of conventional bearingsonly target motion analysis. IEEE Trans. Autom. Control AC-29(9): 775-787

[8] Rao S K 2001 Pseudo-linear estimator for bearings-only passive target tracking. IEE Proc.-Radar Sonar Nav. 148(1): 16-22

[9] Rao S K 2010 Maximum likelihood estimator for bearingsonly passive target tracking in electronic surveillance measure and electronic warfare systems. Defence Sci. J. 60(2): 197-203
[10] Aidala V J and Nardone S C 1982 Biased estimation properties of the pseudolinear tracking filter. IEEE Trans. Aerosp. Electron. Syst. AES-18(4): 432-441

[11] Julier S and Uhlmann J 2004 Unscented filtering and nonlinear estimation. Proc. IEEE 92(3): 401-422

[12] Rao S K, Rajeswari K R and Lingamurty K S 2009 Unscented Kalman filter with application to bearings-only target tracking. IETE J. Res. 55(2): 63-67

[13] Zhang H, Dai G, Sun J and Zhao Y 2013 Unscented Kalman filter and its nonlinear application for tracking a moving target. Optik 124(20): 4468-4471

[14] Rao S K, Kumar D V A N R and Raju K P 2013 Combination of pseudo-linear estimator and modified gain bearings-only extended Kalman filter for passive target tracking in abnormal conditions. Ocean Electron. (SYMPOL) p 3-8

[15] Zhou G, Pelletier M, Kirubarajan T and Quan T 2014 Statically fused converted position and Doppler measurement Kalman Filters. IEEE Trans. Aerosp. Electron. Syst. 50(1): 300-318

[16] Simon D 2006 Optimal state estimation: Kalman, $H_{\infty}$, and nonlinear approaches. Wiley-Interscience, New Jersey

[17] Ristick B, Arulampalam S and Gordon N 2004 Beyond the Kalman filter-particle filters for tracking applications. Artech House and DSTO, London 